\title{
Highly Efficient Multiple Exciton Generation in Colloidal PbSe and PbS Quantum Dots
}

\author{
Randy J. Ellingson, ${ }^{*},+, \|$ Matthew C. Beard, ${ }^{*,+, \|}$ Justin C. Johnson, ${ }^{\ddagger}$ Pingrong Yu, ${ }^{\ddagger}$ \\ Olga I. Micic, ${ }^{\dagger}$ Arthur J. Nozik, ${ }^{*,+, \neq}$ Andrew Shabaev,, ,ll and Alexander L. Efros ${ }^{*, \S}$ \\ Center for Basic Sciences, National Renewable Energy Laborarory, \\ Golden, Colorado 80401, Department of Chemistry, University of Colorado, \\ Boulder, Colorado 80309, and Naval Research Laboratory, Washington, D.C. 20375
}

Received February 10, 2005; Revised Manuscript Received March 19, 2005

\begin{abstract}
We report ultra-efficient multiple exciton generation (MEG) for single photon absorption in colloidal PbSe and PbS quantum dots (QDs). We employ transient absorption spectroscopy and present measurement data acquired for both intraband as well as interband probe energies. Quantum yields of $300 \%$ indicate the creation, on average, of three excitons per absorbed photon for PbSe QDs at photon energies that are four times the QD energy gap. Results indicate that the threshold photon energy for MEG in QDs is twice the lowest exciton absorption energy. We find that the biexciton effect, which shifts the transition energy for absorption of a second photon, influences the early time transient absorption data and may contribute to a modulation observed when probing near the lowest interband transition. We present experimental and theoretical values of the size-dependent interband transition energies for PbSe QDs. We present experimental and theoretical values of the size-dependent interband transition energies for PbSe QDs, and we also introduce a new model for MEG based on the coherent superposition of multiple excitonic states.
\end{abstract}

The formation of multiple electron-hole pairs per absorbed photon in photoexcited semiconductors is a process typically explained by impact ionization (I.I.). In this process, an electron or hole with kinetic energy greater than the semiconductor band gap produces one or more additional electron-hole pairs. The kinetic energy can be created either by applying an electric field or by absorbing a photon with energy above the semiconductor band gap energy. The former is well studied and understood ${ }^{1-3}$ and is the basis for efficient current amplification in avalanche photodiodes. The latter process is less well studied but has been observed in photoexcited $\mathrm{p}-\mathrm{n}$ junctions of $\mathrm{Si}, \mathrm{Ge}$, and $\mathrm{InSb}^{4-7}$

Formation of multiple electron-hole pairs following absorption of a single energetic photon presents a possible route to improved photovoltaic conversion efficiency, because additional photocurrent can be realized while simultaneously bypassing heat generation resulting from phonon scattering. Impact ionization has not, however, contributed meaningfully to improved quantum yield in working solar cells, primarily because the I.I. efficiency does not reach significant values until photon energies reach the ultraviolet

* Corresponding authors. E-mail: randy_ellingson@nrel.gov, matt_beard@nrel.gov, arthur_nozik@nrel.gov, efros@dave.nrl.navy.mil.

$\dagger$ National Renewable Energy Laboratory.

$\doteqdot$ University of Colorado

$\S$ Naval Research Laboratory.

" These authors contributed equally to this work. region of the spectrum. In bulk semiconductors, the threshold photon energy for I.I. exceeds that required for energy conservation alone because, in addition to conserving energy, crystal momentum must be conserved. Additionally, the rate of I.I. must compete with the rate of energy relaxation by electron-phonon scattering. Electron-phonon scattering is a very fast process (sub-ps) in bulk semiconductors, and it has been shown that the rate of I.I. becomes competitive with phonon scattering rates only when the kinetic energy of the electron is many times the band gap energy $\left(\mathrm{E}_{\mathrm{g}}\right) .{ }^{1-3}$ The observed transition between inefficient and efficient I.I. occurs slowly; for example, in Si the I.I. efficiency was found to be only $5 \%$ (i.e., total quantum yield $=105 \%$ ) at $h v \approx 4$ $\mathrm{eV}\left(3.6 \mathrm{E}_{\mathrm{g}}\right)$, and $25 \%$ at $h v \approx 4.8 \mathrm{eV}\left(4.4 \mathrm{E}_{\mathrm{g}}\right){ }^{7,8}$ This large blue-shift of the threshold photon energy for I.I. in semiconductors prevents materials such as $\mathrm{Si}$ and GaAs from yielding improved solar conversion efficiencies. ${ }^{8,9}$

It has recently been proposed, ${ }^{10,11}$ however, that I.I. could be greatly enhanced in semiconductor quantum dots (QDs) (or nanocrystals (NCs)) compared to bulk semiconductors, because the limitations discussed above for I.I. in bulk semiconductors are greatly alleviated in QDs. The rate of electron relaxation through electron-phonon interactions can be significantly reduced because of the discrete character of the electron-hole spectra, and the rate of Auger processes, including the inverse Auger process of impact ionization, is 
greatly enhanced due to carrier confinement and the concomitantly increased electron-hole Coulomb interaction. Additionally, crystal momentum need not be conserved because momentum is not a good quantum number for threedimensionally confined carriers. Indeed, very efficient multiple electron-hole pair (multiexciton) creation by one photon was observed recently in PbSe nanocrystals by Schaller and Klimov. ${ }^{12}$ They reported an excitation energy threshold for the formation of two excitons per photon at $3 E_{g}$, where $E_{g}$ is the absorption energy gap of the nanocrystal (HOMO-LUMO transition energy). The quantum yield (QY) of exciton formation is defined as the average number of ground-state excitons produced per absorbed photon, expressed as a percentage. Schaller and Klimov reported a QY value of $218 \%$ (118\% I.I. efficiency) at $3.8 E_{g}$; QYs above $200 \%$ indicate the formation, on average, of more than two excitons per absorbed photon. The authors explained these results in terms of very efficient noncoherent impact ionization in the nanocrystals by optically excited highly energetic electron-hole pairs.

In this letter, we report a previously unattained QY value of $300 \%$ for $3.9 \mathrm{~nm}$ diameter $\mathrm{PbSe}$ QDs at a photon energy of $4 E_{g}$, indicating the formation, on average, of three excitons per photon for every photoexcited QD in the sample. We provide evidence that the threshold for multiple exciton generation (MEG, an alternate description of impact ionization-see below) by optical excitation is $2 E_{g}$, not $3 E_{g}$ as reported previously for $\mathrm{PbSe} \mathrm{QDs},{ }^{12}$ and we show that comparably efficient MEG occurs also in $\mathrm{PbS}$ nanocrystals. We also introduce a new possible mechanism for MEG, and delineate the unique character of QD electronic structure, which allows for the possibility of a coherent evolution of the quantum state from the initial single exciton to a coherent superposition of multiple exciton states. ${ }^{13}$ A model for MEG in QDs based on the coherent evolution of a single exciton state into multiple exciton states predicts that oscillations (quantum beats) between states of varying numbers of (energy-allowed) exciton states may be observable by ultrafast spectroscopy. ${ }^{13}$ While we have not yet obtained unambiguous experimental evidence for such coherent MEG signature oscillations, we demonstrate and describe how the biexciton effect (also known as the transition Stark shift) ${ }^{14-17}$ produces a probe wavelength-dependent transient absorption that complicates the search for coherent oscillations in the ultrafast lowest-exciton bleach signal.

We have studied three samples of PbSe NCs with diameters ranging from 3.9 to $5.7 \mathrm{~nm}$, and one $\mathrm{PbS} \mathrm{NC}$ sample with a diameter of $5.5 \mathrm{~nm}$. The $\mathrm{PbSe}$ and $\mathrm{PbS} \mathrm{QD}$ samples studied here were obtained from Evident Technologies. The absorption spectrum (Figure 1a) and its second derivative (Figure 1b) at $300 \mathrm{~K}$ for a $5.4 \mathrm{~nm}$ diameter colloidal PbSe QD sample are shown in Figure 1. Minima in the second derivative clearly indicate the optical transition energies. ${ }^{18}$

We have also calculated the size dependence of the transitions between electron and hole levels in PbSe NCs (see Figure 1c) using a theory developed by Kang and Wise, ${ }^{19}$ with modified energy parameters. ${ }^{20}$ Comparison of the theoretical dependence with the experimental data and application of the selection rules $\Delta n=0$ and $\Delta L=0$, allows the identification of the optical transitions (see Figure 1c). Dashed lines in Figures 1c and 1d indicate the splitting by spin-orbit coupling of levels with nonzero angular momentum. ${ }^{19}$ For $5.4 \mathrm{~nm}$ diameter PbSe QDs, the first excitonic peak (Figure 1a and $1 \mathrm{~b}$ ) at $E_{g}=0.76 \mathrm{eV}$ is from the $1 \mathrm{~S}_{\mathrm{h}}-$ $1 \mathrm{~S}_{\mathrm{e}} \mathrm{HOMO}-\mathrm{LUMO}$ transition. A second peak occurs at 0.96 $\mathrm{eV}$ and is assigned to the $1 \mathrm{~S}_{\mathrm{h}}-1 \mathrm{P}_{\mathrm{e}}$ and $1 \mathrm{P}_{\mathrm{h}}-1 \mathrm{~S}_{\mathrm{e}}$ transitions (an exception to $\Delta L=0$ ). ${ }^{21,22}$ The broad shoulder at 1.14 $\mathrm{eV}$ is attributed to the $1 \mathrm{P}_{\mathrm{h}}-1 \mathrm{P}_{\mathrm{e}}$ transition, and the transition at $1.28 \mathrm{eV}$ can be assigned as the weakly allowed $1 \mathrm{~S}_{\mathrm{h}}-2 \mathrm{~S}_{\mathrm{e}}$ (or $2 \mathrm{~S}_{\mathrm{h}}-1 \mathrm{~S}_{\mathrm{e}}$ ) transition (an exception to $\Delta n=0$ ). The peak at $1.52 \mathrm{eV}\left(\sim 2 E_{g}\right)$ can be assigned to the $1 \mathrm{D}_{\mathrm{h}}-1 \mathrm{D}_{\mathrm{e}}$ transition. We assign the next two features between $2 E_{g}$ and $3 E_{g}$ at 1.62 and $1.88 \mathrm{eV}$ to the $2 \mathrm{~S}_{\mathrm{h}}-2 \mathrm{~S}_{\mathrm{e}}$ and $1 \mathrm{~F}_{\mathrm{h}}-1 \mathrm{~F}_{\mathrm{e}}$ transitions, respectively. Finally, the feature at $2.27 \mathrm{eV}$ accompanied by a steep rise in absorbance in this spectral region is assigned to the $2 \mathrm{P}_{\mathrm{h}}-2 \mathrm{P}_{\mathrm{e}}$ transition. One can see that the energy of the $2 \mathrm{P}_{\mathrm{h}}-2 \mathrm{P}_{\mathrm{e}}$ transition is $\sim 3 E_{g}$. We include in the online Supporting Information tabulated results of the theoretical computation of interband transition energies, as a function of size, for PbSe QDs.

We detect multiexcitons by monitoring the signature of multiexciton decay dynamics using transient absorption (TA) spectroscopy; this is the same approach used by Schaller and Klimov. ${ }^{12}$ The magnitude of the photoinduced absorption change at the band edge is proportional to the number of electron-hole pairs created in the sample. The transients are detected by probing either with a band edge (energy gap or HOMO-LUMO transition energy $\equiv E_{g}$ ) probe pulse, or with a mid-IR probe pulse that monitors intraband transitions in the newly created excitons. Although both the band-edge and mid-IR probe signals would incorporate components from excitons with energy above the $1 S_{h}-1 S_{e}$ exciton, our multiple exciton Auger recombination analysis relies only on data for delays $\gtrsim 5 \mathrm{ps}$, by which time carrier multiplication and cooling are complete. Analysis of the QY of multiple exciton generation requires careful knowledge of the initial excitation levels within the sample, and of multiexciton Auger recombination dynamics. The average number of photons absorbed per QD follows Poisson statistics such that $N_{\mathrm{eh}}=J_{\mathrm{p}} \sigma_{\mathrm{a}}$, where $J_{\mathrm{p}}$ is the photon fluence and $\sigma_{\mathrm{a}}$ is the absorption cross section at the excitation photon energy. ${ }^{23}$ To study MEG resulting from absorption of a single photon, we maintain a constant $N_{\text {eh }}$ value ensuring that the vast majority of photoexcited QDs have absorbed just one photon (e.g., for $N_{\text {eh }}=0.25,<14 \%$ of photoexcited QDs absorb more than one photon). The details describing how we determine the MEG QYs from the TA data are presented in the online Supporting Information.

Figure 2a shows the exciton population (determined from the mid-IR absorption) dynamics for a $5.7 \mathrm{~nm}$ diameter sample $\left(E_{g}=0.72 \mathrm{eV}\right.$, and $\left.N_{\mathrm{eh}}=0.25\right)$ while varying the ratio of the energy of the pump photon to that of the band gap $\left(E_{h v} / E_{g}\right)$ from 1.9 to 5.0. The increasingly prominent decay seen in Figure 2a as the photon energy increases is associated with nonradiative recombination of multiexcitons 

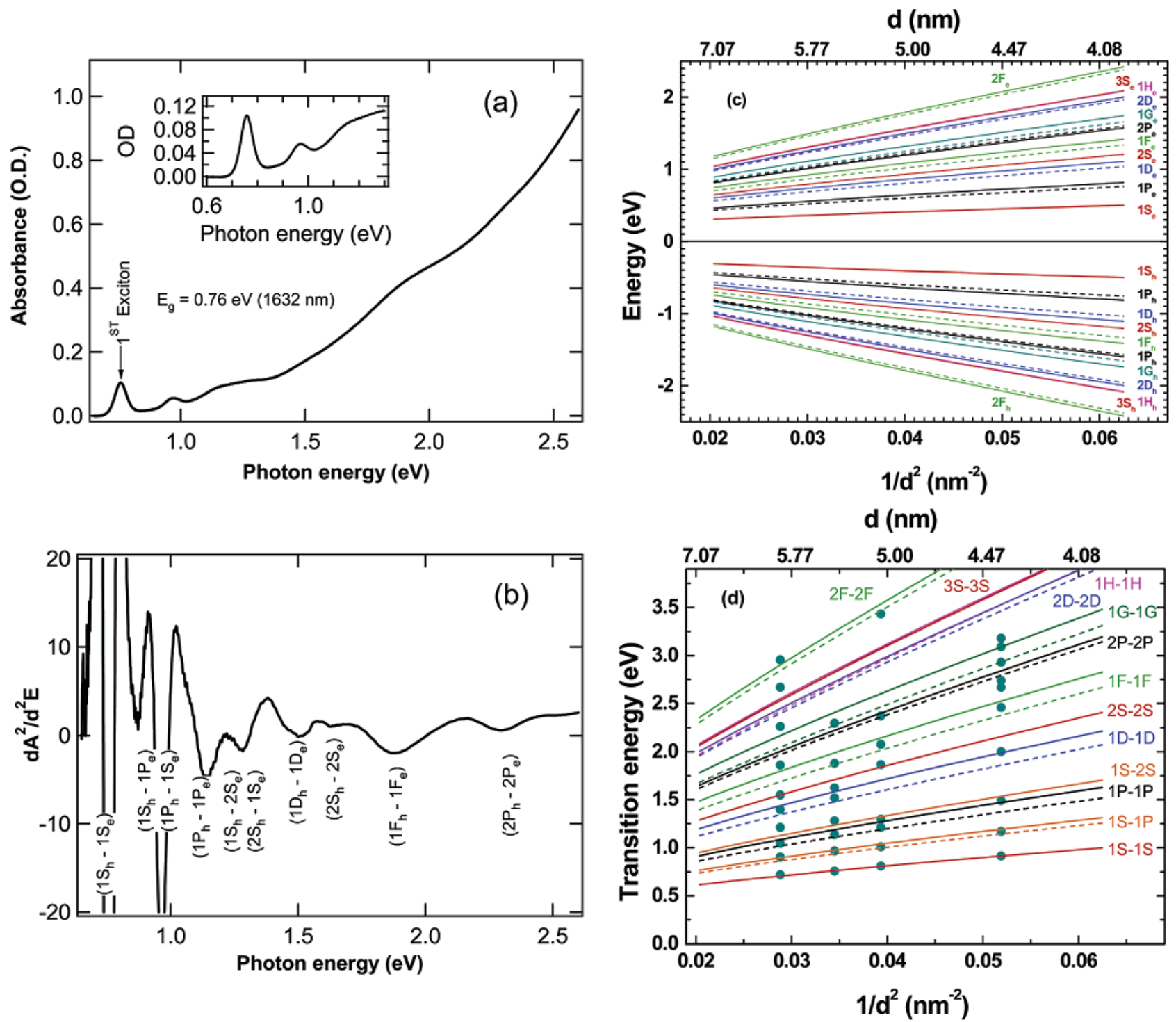

Figure 1. (a) Absorption spectrum and (b) its second derivative of $5.4 \mathrm{~nm} \mathrm{PbSe}$ QDs. Transitions at higher energies $(1.1 \mathrm{eV}<h v<3.0$ $\mathrm{eV}$ ) are obtained from the second derivative of the rising absorption spectrum in this region (not shown). The inset of (a) shows detail of the absorption spectrum for the region including the $\left(1 \mathrm{~S}_{\mathrm{h}}-1 \mathrm{~S}_{\mathrm{e}}\right),\left(1 \mathrm{~S}_{\mathrm{h}}-1 \mathrm{P}_{\mathrm{e}}\right) /\left(1 \mathrm{P}_{\mathrm{h}}-1 \mathrm{~S}_{\mathrm{e}}\right)$, and $\left(1 \mathrm{P}_{\mathrm{h}}-1 \mathrm{P}_{\mathrm{e}}\right)$ interband transitions. (c) Theoretical size dependence of the energy level structure in PbSe QDs. (d) Theoretical transitions for four QD sizes with experimental transitions superimposed. The typical error for the experimental peaks is $\pm 0.05 \mathrm{eV}$. Dashed lines in (c) and (d) show spin-orbital splitting of those states with angular momentum greater than zero.

via Auger recombination. ${ }^{24}$ Multiple excitons within a nanocrystal undergo Auger recombination at rates that increase with the number of excitons per QD. Our PbSe QD samples exhibit single exciton lifetimes ( $\tau_{1} \gtrsim 6000 \mathrm{ps}$ ) much longer than their biexciton lifetimes $\left(\tau_{2} \lesssim 100 \mathrm{ps}\right)$; a large ratio $\tau_{1} / \tau_{2}$ greatly facilitates analysis of multiexciton decay dynamics. Maintaining a constant excitation level per QD (photons absorbed per QD), we compare the amplitudes of the fast decay components as a function of pump photon energy. The analysis includes data for delays beyond the TA signal rise time. The density-dependent carrier dynamics of the signal decay provide a quantitative measurement of the number of electron-hole pairs at the absorption band edge per absorbed photon, and thus of the QY. Generation of additional excitons (MEG) occurs within the rise time of the signal. The advantage to probing the intraband photoinduced absorption in the infrared rather than probing the bleach at the band edge energy, is that the detected transients are linear to high exciton number; however, the results were independent of the probe wavelength regime. The experimental apparatus has been described elsewhere. ${ }^{25}$

The dependence of the MEG QY on the ratio of the pump photon energy to the band gap $\left(E_{h v} / E_{g}\right.$ varied from 1.9 to 5.0) for $\mathrm{PbSe} \mathrm{QD}$ samples with $E_{g}=0.72 \mathrm{eV}$ (dia. $=5.7$ $\mathrm{nm}), E_{g}=0.82 \mathrm{eV}($ dia. $=4.7 \mathrm{~nm})$, and $E_{g}=0.91 \mathrm{eV}$ (dia. $=3.9 \mathrm{~nm}$ ) is shown in Figure 2b. For all three samples, the sharp rise in QY begins at about three times the energy gap, a result in agreement with that reported by Schaller and Klimov. ${ }^{12}$ Our data show that for the $3.9 \mathrm{~nm}$ QD $\left(E_{g}=0.91\right.$ $\mathrm{eV})$, the QY reaches a remarkable value of $300 \%$ at $E_{h v} / E_{g}$ $=4.0$, indicating that on average the QDs produce three excitons per absorbed photon. For the other two $\mathrm{PbSe}$ samples $\left(E_{g}=0.82 \mathrm{eV}(4.7 \mathrm{~nm}\right.$ dia. $)$ and $0.72 \mathrm{eV}(5.7 \mathrm{~nm}$ dia.)), it is estimated that a QY of $300 \%$ is reached at an 

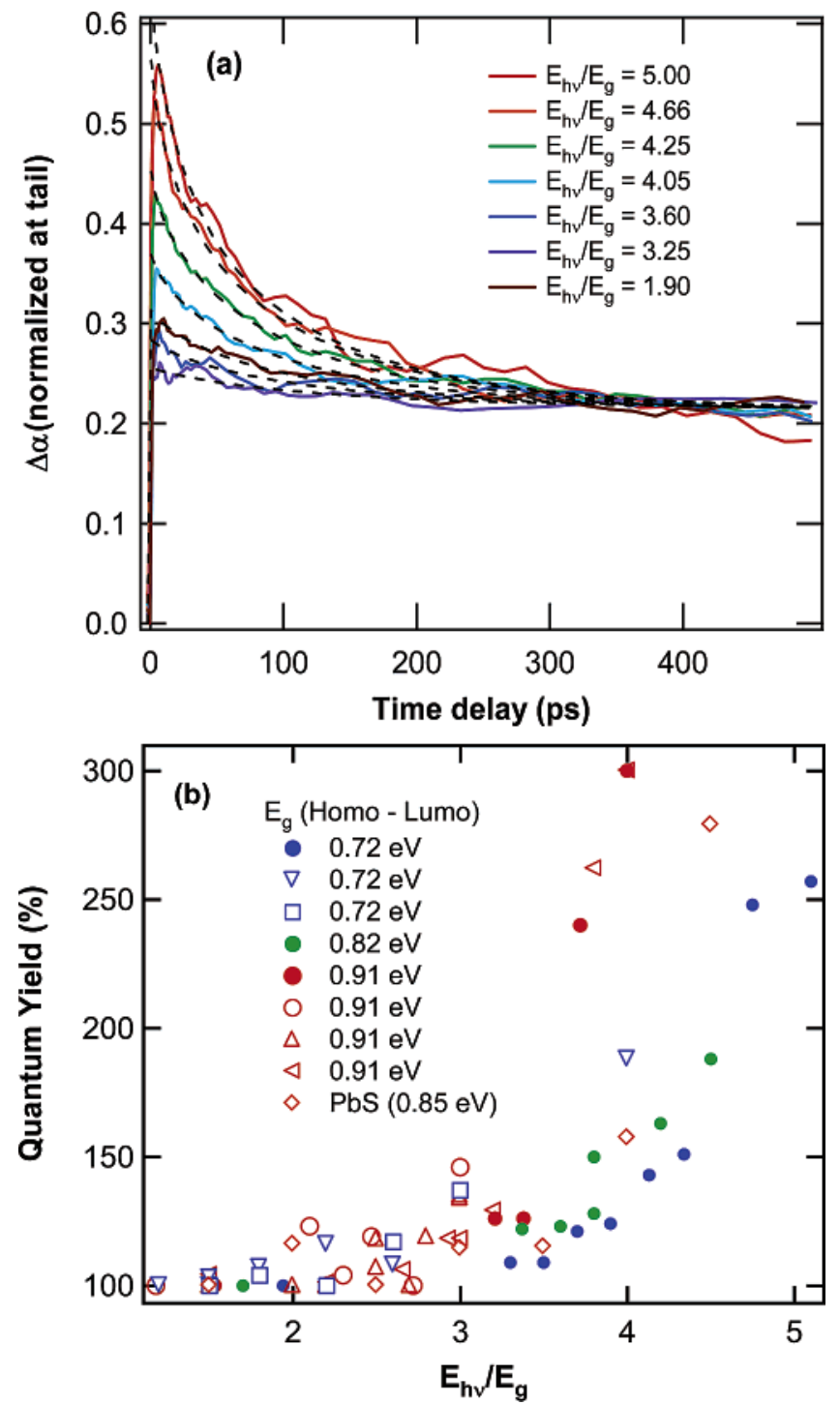

Figure 2. (a) Exciton population decay dynamics obtained by probing intraband (intraexciton) transitions in the mid-IR at 5.0 $\mu \mathrm{m}$ for a sample of $5.7 \mathrm{~nm}$ diameter PbSe QDs. (b) QY for exciton formation from a single photon vs photon energy expressed as the ratio of the photon energy to the QD band gap (HOMO-LUMO energy) for three PbSe QD sizes and one $\mathrm{PbS}$ (dia. = 3.9, 4.7, 5.4, and $5.5 \mathrm{~nm}$, respectively, and $E_{g}=0.91,0.82,0.73 \mathrm{eV}$, and 0.85 $\mathrm{eV}$ respectively). Solid symbols indicate data acquired using midinfrared probe; open symbols indicate band-edge probe energy. Quantum yield results were independent of the probe energy utilized.

$E_{h v} / E_{g}$ value of 5.5 . Note that the $2 \mathrm{P}_{\mathrm{h}}-2 \mathrm{P}_{\mathrm{e}}$ transition is resonant with $3 \mathrm{E}_{\mathrm{g}}$ excitation, corresponding to the sharp onset of increased MEG efficiency. If this symmetric transition $\left(2 \mathrm{P}_{\mathrm{h}}-2 \mathrm{P}_{\mathrm{e}}\right)$ dominates the absorption at $\sim 3 \mathrm{E}_{\mathrm{g}}$, the resulting excited state provides both the electron and the hole with excess energy of $1 \mathrm{E}_{\mathrm{g}}$, in resonance with the lowest exciton absorption (at $1 \mathrm{E}_{\mathrm{g}}$ ).

Our data also show that the QY begins to surpass 100\% at $E_{h v} / E_{g}$ values greater than 2.0 (see Figure $2 \mathrm{~b}$ ). We have carefully measured $16 \mathrm{QY}$ values between $2.1 \mathrm{E}_{\mathrm{g}}$ and $2.9 \mathrm{E}_{\mathrm{g}}$ (mean value $=109.8 \%)$ and $11 \mathrm{QY}$ values between $1.2 \mathrm{E}_{\mathrm{g}}$ and $2.0 \mathrm{E}_{\mathrm{g}}$ (mean value $=101.3 \%$ ). Application of statistical $t$-tests show that the QY values for photon energies between
$1 \mathrm{E}_{\mathrm{g}}$ and $2 \mathrm{E}_{\mathrm{g}}$ are not statistically different from $100 \%(P$ value $=0.105$ ), while the difference in QYs between $1.2 \mathrm{E}_{\mathrm{g}}-2.0 \mathrm{E}_{\mathrm{g}}$ and $2.1 \mathrm{E}_{\mathrm{g}}-2.9 \mathrm{E}_{\mathrm{g}}$ is very statistically significant with a $P$ value of 0.001 . Simple visual inspection of Figure $2 b$ indicates a significant difference between the QY values between $1 \mathrm{E}_{\mathrm{g}}-2 \mathrm{E}_{\mathrm{g}}$ and $2 \mathrm{E}_{\mathrm{g}}-3 \mathrm{E}_{\mathrm{g}}$.

A photon with energy above $2 \mathrm{E}_{\mathrm{g}}$ creates in a nanocrystal a coherent superposition of single and various multiexciton states, which are coupled by the multielectron Coulomb interaction. ${ }^{13}$ This is a result of the degeneracy of an electron (hole) with kinetic energy larger than $\mathrm{E}_{\mathrm{g}}$ with a multiparticle state consisting of two electrons and a hole (two holes and an electron) occupying the lowest possible levels of the conduction and valence bands. In the simplest case of the $2 \mathrm{P}_{\mathrm{e}}$ electron $\left(2 \mathrm{P}_{\mathrm{h}}\right.$ hole $)$ state in $\mathrm{PbSe}$ QDs, the multiparticle state is the $1 \mathrm{~S}_{\mathrm{e}} 1 \mathrm{~S}_{\mathrm{e}} 1 \mathrm{~S}_{\mathrm{h}}\left(1 \mathrm{~S}_{\mathrm{h}} 1 \mathrm{~S}_{\mathrm{h}} 1 \mathrm{~S}_{\mathrm{e}}\right)$ electron-hole configuration. This $1 \mathrm{~S}_{\mathrm{e}} 1 \mathrm{~S}_{\mathrm{e}} 1 \mathrm{~S}_{\mathrm{h}}\left(1 \mathrm{~S}_{\mathrm{h}} 1 \mathrm{~S}_{\mathrm{h}} 1 \mathrm{~S}_{\mathrm{e}}\right)$ electron-hole configuration is coupled with the single particle $2 \mathrm{P}_{\mathrm{e}}$ electron $\left(2 \mathrm{P}_{\mathrm{h}}\right.$ hole) state via a direct multielectron Coulomb interaction. The same type of Coulomb coupling leads to nonradiative Auger recombination of a photoexcited electron-hole pair in a nanocrystal with an extra carrier-the process that is the inverse of direct impact ionization and which has been considered in ref 26 . As a result, a photon with energy above $3 \mathrm{E}_{\mathrm{g}}$ directly creates multiexciton states. In the case of excitation in the energy interval between $2 \mathrm{E}_{\mathrm{g}}$ and $3 \mathrm{E}_{\mathrm{g}}$, direct Coulomb interaction couples a symmetric electron-hole pair created by light to an asymmetric pair where the total kinetic energy is resident in one carrier and is sufficient to create a multiparticle electron-hole configuration.

The time scale for the formation of the multiexciton state is inversely proportional to the strength of the Coulomb coupling. Calculation of the Coulomb matrix element between single and multiexciton states similar to that presented in ref 26 has shown that photons with energy $\sim 3 \mathrm{E}_{\mathrm{g}}$, exciting $2 \mathrm{P}_{\mathrm{e}}-2 \mathrm{P}_{\mathrm{h}}$ transitions in $4.7 \mathrm{~nm}$ diameter $\mathrm{PbSe}$ QDs, create multiexciton states within as little as $250 \mathrm{fs}$. The oscillation rate between single and multiexciton states created by the absorbed photon occurs on the same time scale. Note that this time competes favorably with the cooling time of a few ps that we observe, an indication that very fast cooling of the single exciton is unlikely to prevent MEG. As a result, in PbSe NCs cooling occurs via MEG and energy is lost via dephasing of the coherent multiexciton state by phonon emission (see Figure 3).

This idea differs from the previous explanation of incoherent I.I. where the rate of I.I. competes with the rate of phonon emission. In the model of incoherent I.I., phonon-based cooling is an independent parallel process unrelated to the process of exciton multiplication. A previous theoretical calculation of carrier multiplication in CdSe QDs was based on incoherent I.I. ${ }^{27}$ In the coherent MEG model, the creation of the multiple exciton state from one exciton occurs as a natural consequence of the quantum mechanics. The efficiency of exciton multiplication in this model, however, is determined by the ratio of the rates with which single and multiexciton coupled states (of varying exciton number) within the coherent superposition undergo dephasing. This 


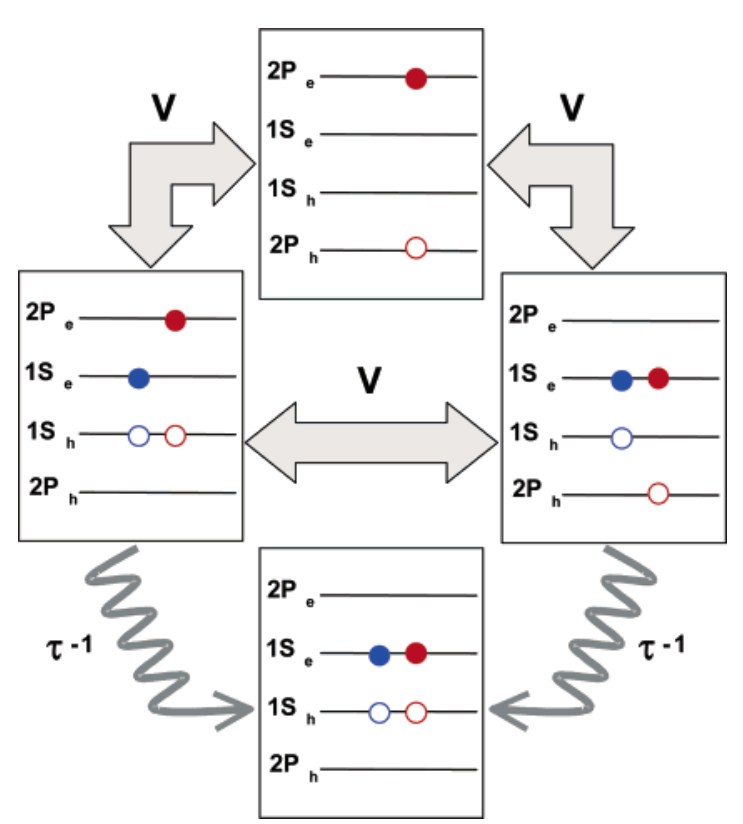

Figure 3. Photoexcitation at $3 \mathrm{E}_{\mathrm{g}}$ creates a $2 \mathrm{P}_{\mathrm{e}}-2 \mathrm{P}_{\mathrm{h}}$ exciton state. This state is coupled to multiparticle states with matrix element V and forms a coherent superposition of single and multiparticle exciton states within $\sim 250$ fs. The coherent superposition dephases due to interactions with phonons; asymmetric states (such as a $2 \mathrm{P}_{\mathrm{e}}-$ $1 \mathrm{~S}_{\mathrm{h}}$ ) couple strongly to LO phonons and dephase at a rate of $\tau^{-1}$.

ratio determines, on average, the number of excitons that survive. The evident disparity between the initial rate of exciton multiplication and the eventual rate of Auger recombination indicates that the single and multiple exciton states are coherently coupled. Auger recombination occurs on a much slower time scale, strongly suggesting that this inherent irreversibility between the coherent and incoherent regimes allows high QYs for MEG.

The fundamental electronic properties of $\mathrm{PbSe}$ and $\mathrm{PbS}$ QDs present a scenario of particular interest for multiple exciton generation. Phonon scattering serves as the primary mechanism for carrier cooling to the band-edge states in bulk and nanoscale semiconductors. Quantum dots in the strong confinement regime $\left(d \ll a_{\mathrm{e}, \text { Bohr }}, d \ll a_{\mathrm{h} \text {,Bohr }}\right)$ exhibit sparse electronic states, the intraband spacing of which increases with decreasing effective mass. The interlevel spacing for electrons and holes can easily reach several times the optical phonon energy, reducing the efficiency of phonon emission as a relaxation mechanism; this effect is known as the phonon bottleneck. Frequently, however, semiconductor QDs exhibit conduction band energy level spacing much larger than that of the valence band, due to the smaller electron effective mass, $m_{\mathrm{e}}{ }^{*}$. For such semiconductors with $m_{\mathrm{e}}{ }^{*} \ll m_{\mathrm{h}}{ }^{*}$, Augerlike cooling provides the relaxation route to bypass any phonon bottleneck. ${ }^{28,29}$ Inhibiting Auger-like cooling by removing or eliminating one of the carriers required for the Auger process has been shown to result in an approximate 10-fold decrease in the relaxation rate in QDs of CdSe ${ }^{16,17,30}$ and InP. ${ }^{31}$ However, for $\mathrm{PbSe}$ and $\mathrm{PbS}$, the electron and hole effective masses are both (1) relatively small and (2) essentially identical. These two material characteristics combine to provide a situation in which the intraband level spacings for both electrons and holes are as many as 10 or more multiples of the LO phonon energy $(17 \mathrm{meV})^{32}$ and the process of Auger-like cooling thus cannot facilitate an increased cooling rate. The scenario for a significant phonon bottleneck and an absence of Auger-like cooling favors relatively efficient MEG, as observed for photon energies $\approx 3 \mathrm{E}_{\mathrm{g}}$.

For photon energies between $2 \mathrm{E}_{\mathrm{g}}$ and $3 \mathrm{E}_{\mathrm{g}}$, the efficiency of MEG is clearly low: we note that the mean QY between 2.1 and $2.9 \mathrm{E}_{\mathrm{g}}$ is only $\sim 110 \%$. Formally allowed interband transitions are "symmetric", between valence and conduction states of identical envelope angular momenta (e.g., $1 \mathrm{~S}_{\mathrm{h}}-$ $1 \mathrm{~S}_{\mathrm{e}}$, or $1 \mathrm{P}_{\mathrm{h}}-1 \mathrm{P}_{\mathrm{e}}$ ). However, an unexpectedly strong asymmetric interband transition involving the $1 \mathrm{~S}$ and $1 \mathrm{P}$ states $\left(1 \mathrm{~S}_{\mathrm{h}}-1 \mathrm{P}_{\mathrm{e}}\right.$ and $1 \mathrm{P}_{\mathrm{h}}-1 \mathrm{~S}_{\mathrm{e}}$ transitions) occurs in $\mathrm{PbSe} \mathrm{QDs},{ }^{19,22,33,34}$ a result also shown clearly in our PbSe absorption spectra (see, e.g., Figure 1a) as well as for PbS. ${ }^{19,35,36}$ This suggests that asymmetric transitions above the $1 \mathrm{~S}-1 \mathrm{P}$ transition may also have significant oscillator strength. In the pump photon energy range of $2 E_{\mathrm{g}}-3 \mathrm{E}_{\mathrm{g}}$, asymmetric states for which either the electron or the hole resides in the $1 \mathrm{~S}$ level allow the other carrier sufficient energy to accommodate generation of an additional exciton. The Fröhlich interaction of electrons (holes) with polar phonons is the strongest mechanism for electron (hole)-phonon coupling in nanocrystals. The magnitude of this coupling increases inversely proportional to the nanocrystal radius $^{37,38}$ and polar phonons should be responsible for carrier relaxation in nanocrystals. The interaction of the polar phonons with the electron-hole pair, however, is determined by the total charge distribution of this pair within the nanocrystals. ${ }^{39}$ For example, in a semiconductor with simple parabolic conduction and valence bands, an allowed optical excitation creates electron-hole pairs or excitons that do not interact strongly with phonons, ${ }^{40}$ because for such bands, photoexcitation creates the electron and hole in states with the same quantum numbers and the charge distributions of the carriers exactly compensate each other at each point in the nanocrystal. This remains true for $\mathrm{PbSe}$ and $\mathrm{PbS}$ nanocrystals, although their conduction and valence bands are not parabolic: the conduction and valence bands in these materials are nearly identical, and when optical excitation creates an electron-hole pair with the same quantum numbers, the interaction of this pair with a polar phonon should be extremely weak. However, this is not the case for asymmetric electron-hole states; in these cases, the exciton-phonon coupling can be very strong leading to fast dephasing and carrier relaxation. Our MEG data, showing only low efficiencies below $3 \mathrm{E}_{\mathrm{g}}$, concur with the prospect of an enhanced cooling rate reducing the QY for asymmetric states (Figure 2b). For photon energies exceeding $3 E_{g}$, we correlate the increasing efficiency of MEG with an increase in the density of levels accessible by single- and multiexciton states. Our data indicate that phonon interaction with the coherent quantum state occurs during dephasing, a process which allows the ultimate survival of a multiple exciton state. Even at the most efficient MEG observed, absorption of a $4 \mathrm{E}_{\mathrm{g}}$ photon results in an exciton QY of $300 \%$ indicating that on average we generate $1 \mathrm{E}_{\mathrm{g}}$ of thermal energy (the equivalent of $>50$ optical phonons) during the MEG dephasing process. 

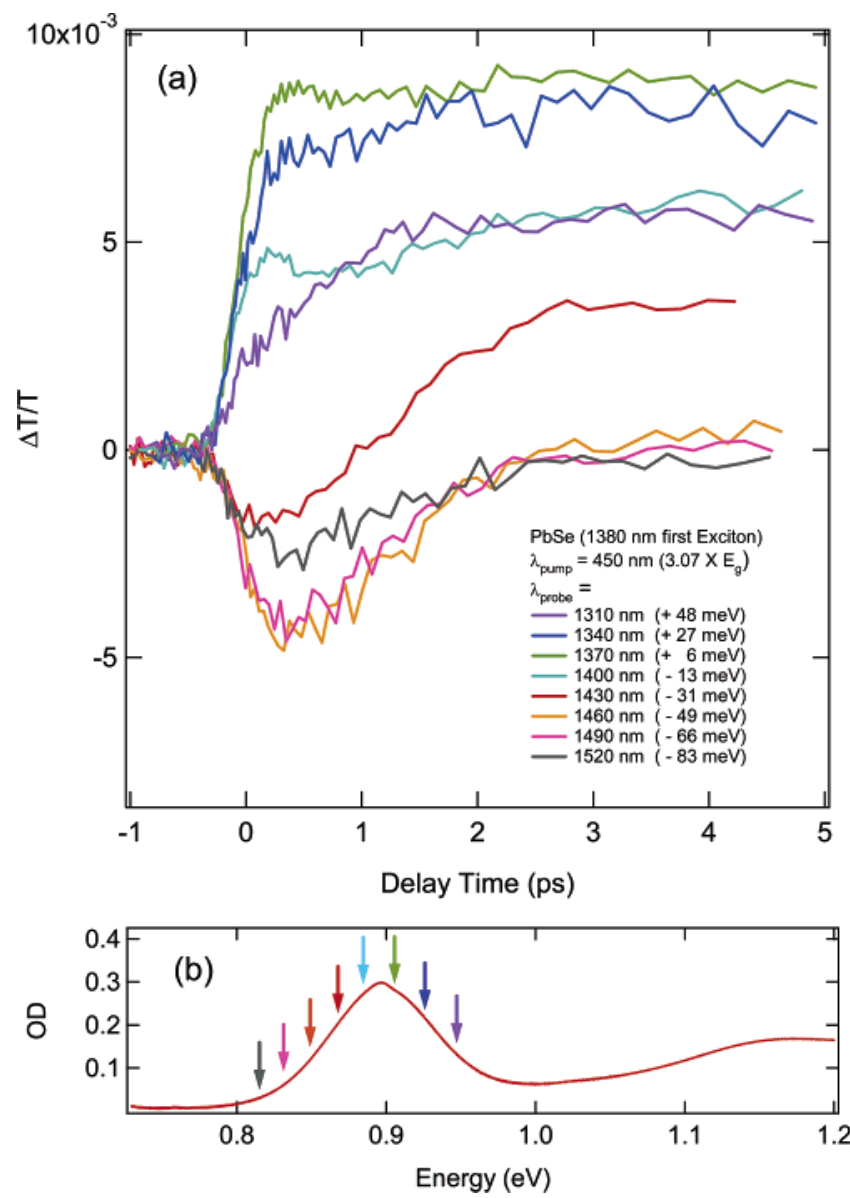

Figure 4. (a) Early-time transient absorption data for a $3.9 \mathrm{~nm}$ diameter $\mathrm{PbSe} \mathrm{QD}$ sample, acquired at various probe energies (shown in (b)), near the $1 \mathrm{~S}$ exciton energy.

While investigating MEG efficiencies, we also studied the rise time of the TA data to search for signatures of the actual multiplication process. Although we have not yet observed an oscillatory behavior (quantum beats) that we can ascribe to MEG, we do observe a modulation in the early time data when probing at, or slightly to the red of, the $1 S_{h}-1 S_{e}$ TA bleach peak. Figure 4a shows the probe wavelength dependence on the early time photoinduced bleach dynamics, for excitation at slightly greater than $3 \mathrm{E}_{\mathrm{g}}$. The arrows in Figure $4 \mathrm{~b}$ indicate the positions of the probe wavelengths relative to the linear absorption spectrum at the $1 \mathrm{~S}_{\mathrm{h}}-1 \mathrm{~S}_{\mathrm{e}}$ transition. Probing $\sim 27 \mathrm{meV}$ above the peak (dark blue), the rise consists of a very fast component followed by a slower but essentially smooth component. Probing near to the peak (green) and slightly below the peak (light blue), the rise shows a modulation with a peak followed by a shallow dip within the first few hundred fs, and then a steady rise over the subsequent $\sim 2$ ps. Probing further to the red, a clear dip develops, indicating a short-live photoinduced absorption (PA) with a rapid onset followed by a decay until $\sim 3$ ps. The modulation appears, therefore, to consist of the superposition of a PA signal and a state-filling-induced bleach; this shape can be generated either by a short-lived PA summed with a smooth rise, or by a short-lived bleach (on the high-energy side of the peak) summed with the same smooth rise. Such derivative-like behavior around the peak

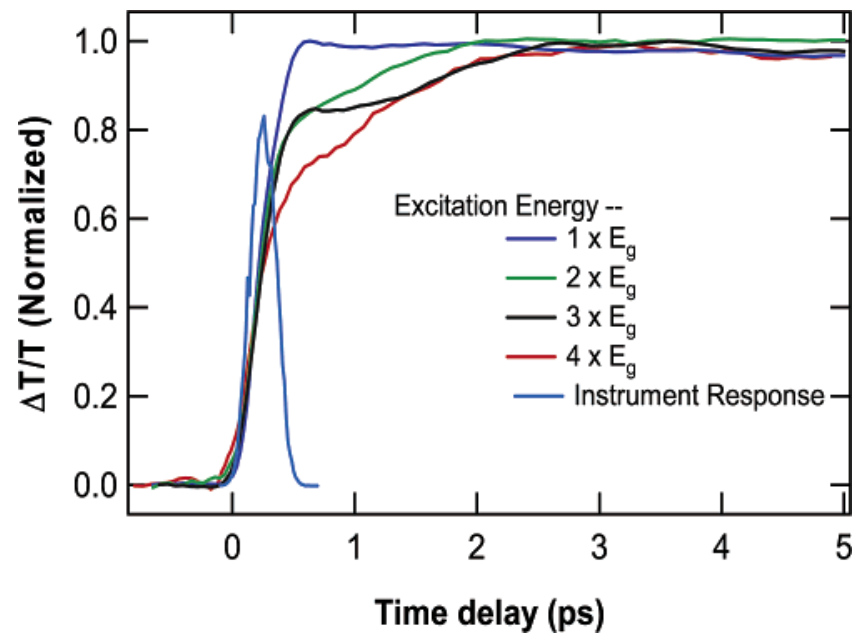

Figure 5. Rise time dynamics probing the bleach of the lowest $1 \mathrm{~S}_{\mathrm{h}}-1 \mathrm{~S}_{\mathrm{e}}$ (HOMO-LUMO) excitation transition after pumping at various photon energies for the $3.9 \mathrm{~nm}$ diameter $\mathrm{PbSe} \mathrm{QD}$ sample. A representative cross-correlation between the pump and probe pulses is also displayed.

of the $1 \mathrm{~S}$ bleach indicates the influence of the biexciton effect, whereby the initial photoexcitation induces a red-shift in the absorbing state seen by the probe photon. As previously observed for CdSe QDs, ${ }^{17}$ the recovery of the PA observed at red-shifted probe wavelengths correlates to the cooling time from higher excited states to the $1 \mathrm{~S}_{\mathrm{h}}-1 \mathrm{~S}_{\mathrm{e}}$ state; thus, the biexciton effect appears to be strongest for higher excited states than for completely relaxed band-edge excitons. The biexciton effect obscures the pure state-filling dynamics, and thus may complicate the direct observation of the quantum beating predicted by the coherent superposition model. In addition, intraband transitions between $1 \mathrm{~S}_{\mathrm{e}}$ and $2 \mathrm{P}_{\mathrm{e}}$ (or $1 \mathrm{~S}_{\mathrm{h}}$ and $\left.2 \mathrm{P}_{\mathrm{h}}\right)$ also complicate the analysis. Further measurements are in progress to fully characterize the biexciton effect and explore its dependence (if any) on MEG efficiency.

Figure 5 shows the excitation energy dependence of the normalized early time band edge TA for the photon energies of $1,2,3$, and $4 \mathrm{E}_{\mathrm{g}}$. The TA bleach rises quickly and completely for $1 \mathrm{E}_{\mathrm{g}}$ excitation, in accord with the instantaneous populating of the $1 S_{\mathrm{h}}-1 S_{\mathrm{e}}$ exciton state. For higher photon energies, the rise time exhibits a slower component, which we correlate to the time required for complete cooling of the carriers. The traces at $3 \mathrm{E}_{\mathrm{g}}$ and $4 \mathrm{E}_{\mathrm{g}}$ show indications of a modulated signal in the first $\sim 2 \mathrm{ps}$, as shown also in Figure $4 \mathrm{~b}$ for the traces at, and slightly red-shifted from, the $1 \mathrm{~S}_{\mathrm{h}}-1 \mathrm{~S}_{\mathrm{e}}$ exciton peak. The largest MEG QY occurs here for $4 \mathrm{E}_{\mathrm{g}}$, though the modulation is less noticeable than for $3 \mathrm{E}_{\mathrm{g}}$ excitation. Note that these data were acquired at the first exciton peak as opposed to slightly lower energy where biexciton effects may reveal deeper modulation. For $2 \mathrm{E}_{\mathrm{g}}$ excitation shown in Figure 5 we observe a slow component to the rise time, with the TA signal reaching the peak value at $\sim 2.5 \mathrm{ps}$. In the case of $\mathrm{PbSe}$ and $\mathrm{PbS}$, where the density of states for electrons and holes are equally sparse, a slow cooling rate can be expected. The biexciton effect does not influence the observed MEG efficiency results, which are based on an analysis of scaled dynamics and not absolute 
magnitudes. ${ }^{12}$ Utilizing an infrared probe near the intraband $1 \mathrm{~S}_{\mathrm{e}}-1 \mathrm{P}_{\mathrm{e}}\left(\right.$ or $1 \mathrm{~S}_{\mathrm{h}}-1 \mathrm{P}_{\mathrm{h}}$ ) transition energy, we have conducted preliminary measurements of the rise time for pump photon energies producing multiple excitons. In these data, we observe a relatively slow $(\sim 2 \mathrm{ps})$ and featureless rise time, and no sign of biexciton effect. Analysis of subsequent Auger recombination decay data for either interband (band edge) or intraband TA concur, indicating that the biexciton effect, though influential in the first few ps of the TA signal, does not affect the long-term decay dynamics.

The work reported here provides a confirmation of the previous report of efficient MEG in PbSe. We observed a previously unattained $300 \%$ QY exciting at $4 \mathrm{E}_{\mathrm{g}}$ in $\mathrm{PbSe}$ QDs, indicating that we generate an average of three excitons per photon absorbed. In addition, we present the first known report of multiple exciton generation in $\mathrm{PbS} \mathrm{QDs}$, at an efficiency comparable to that in PbSe QDs. We have shown that a single photon with energy larger than $2 E_{g}$ can generate multiple excitons in PbSe nanocrystals, and we introduce a new model for MEG based on the coherent superposition of multiple excitonic states. Multiple exciton generation in colloidal QDs represents a new and important mechanism that may greatly increase the conversion efficiency of solar cell devices.

Acknowledgment. R.J.E., M.C.B., P.Y., J.C.J., O.I.M., and A.J.N. were supported by the U.S. Department of Energy, Office of Science, Office of Basic Energy Sciences, Division of Chemical Sciences, Geosciences, and Biosciences. A.S. and A.L.E. were supported by the Office of Naval Research and the Photovoltaics Program of the U.S. Department of Energy, Office of Energy Efficiency and Renewable Energy. Also, A.S. thanks the National Research Council for fellowship support, and A.J.N. thanks Eva NozikGrayck for discussions on statistics. We thank Evident Technologies for contributing QD samples.

Supporting Information Available: Discussion of Auger recombination of multiple excitons generated by direct excitation, description of the analytical approach used to determine multiple exciton generation efficiencies, and tabular values (Excel format) of interband transition energies for $\mathrm{PbSe}$ QDs. This material is available free of charge via the Internet at http://pubs.acs.org.

\section{References}

(1) Jung, H. K.; Taniguchi, K.; Hamaguchi, C. J. Appl. Phys. 1996, 79, 2473.

(2) Harrison, D.; Abram, R. A.; Brand, S. J. Appl. Phys. 1999, 85, 8186.

(3) Bude, J.; Hess, K. J. Appl. Phys. 1992, 72, 3554.

(4) Vavilov, V. S. J. Phys. Chem. Solids 1959, 8, 223.

(5) Tauc, J. J. Phys. Chem. Solids 1959, 8, 219.

(6) Hodgkinson, R. J. Proc. Phys. Soc. 1963, 82, 1010

(7) Christensen, O. J. Appl. Phys. 1976, 47, 690.

(8) Wolf, M.; Brendel, R.; Werner, J. H.; Queisser, H. J. J. Appl. Phys. 1998, 83, 4213
(9) Kolodinski, S.; Werner, J. H.; Wittchen, T.; Queisser, H. J. Appl. Phys. Lett. 1993, 63, 2405.

(10) Nozik, A. J. Physica E 2002, 14, 115

(11) Nozik, A. J. Annu. Rev. Phys. Chem. 2001, 52, 193.

(12) Schaller, R.; Klimov, V. Phys. Rev. Lett. 2004, 92, 186601.

(13) Shabaev, A.; Efros, A. L., to be published.

(14) Hu, Y. Z.; Koch, S. W.; Lindberg, M.; Peyghambarian, N.; Pollock, E. L.; Abraham, F. F. Phys. Rev. Lett. 1990, 64, 1805.

(15) Klimov, V.; Hunsche, S.; Kurz, H. Phys. Rev. B 1994, 50, 81108113.

(16) Klimov, V. I.; Mikhailovsky, A. A.; McBranch, D. W.; Leatherdale, C. A.; Bawendi, M. G. Phys. Rev. B 2000, 61, R13349.

(17) Klimov, V. I. J. Phys. Chem. B 2000, 104, 6112.

(18) Fernee, M. J.; Watt, A.; Warner, J.; Cooper, S.; Heckenberg, N.; Rubinsztein-Dunlop, H. Nanotechnology 2003, 14, 991.

(19) Kang, I.; Wise, F. W. J. Opt. Soc. Am. B 1997, 14, 1632.

(20) We describe our absorption spectra assuming a complete symmetry of the conduction and valence with remote band contribution to the electron and hole masses $m_{0} / m_{\mathrm{e}}=m_{0} / m_{\mathrm{h}}=1.5$ and Kane energy parameter $2 \mathrm{P}^{2} / m=3.15 \mathrm{eV}$ and bulk energy gap $\mathrm{E}_{\mathrm{g}}=0.28 \mathrm{eV}$ (see ref 19).

(21) The $1 \mathrm{~S}-1 \mathrm{P}$ transitions involve a change of parity ( $\mathrm{S}$ to $\mathrm{P}$ and vice versa) and are not allowed formally. Its appearance was interpreted as a result of reduced symmetry of the nanocrystal surface or the existence of electric fields from charge trapped in surface states (see ref 22).

(22) Wehrenberg, B. L.; Wang, C.; Guyot-Sionnest, P. J. Phys. Chem. B 2002, 106, 10634.

(23) Klimov, V. I.; Mikhailovsky, A. A.; McBranch, D. W.; Leatherdale, C. A.; Bawendi, M. G. Science 2000, 287, 1011.

(24) Klimov, V. I.; Mikhailovsky, A. A.; Xu, S.; Malko, A.; Hollingsworth, J. A.; Leatherdale, C. A.; Eisler, H.-J.; Bawendi, M. G. Science 2000, 290, 314.

(25) Ellingson, R. J.; Blackburn, J. L.; Yu, P.; Rumbles, G.; Micic, O. I.; Nozik, A. J. J. Phys. Chem. B 2002, 106, 7758.

(26) Chepic, D. I.; Efros, A. L.; Ekimov, A. I.; Ivanov, M. G.; Kharchenko, V. A.; Kudriavtsev, I. A.; Yazeva, T. V. J. Lumin. 1990, 47, 113.

(27) Califano, M.; Zunger, A.; Franceschetti, A. Appl. Phys. Lett. 2004, 84, 2409.

(28) Auger-like cooling occurs when, for example, the electron transfers its excess energy to the hole through a Coulomb interaction and the hole subsequently cools, by phonon scattering, through the higher density of states in the valence band.

(29) Efros, A. L.; Kharchenko, V. A.; Rosen, M. Solid State Commun. 1995, 93, 281.

(30) Guyot-Sionnest, P.; Shim, M.; Matranga, C.; Hines, M. Phys. Rev. B 1999, 60, R2181.

(31) Blackburn, J. L.; Ellingson, R. J.; Micic, O. I.; Nozik, A. J. J. Phys. Chem. B 2003, 107, 102.

(32) Dalven, R. Solid State Phys. 1973, 28, 179.

(33) Murray, C. B.; Sun, S. H.; Gaschler, W.; Doyle, H.; Betley, T. A.; Kagan, C. R. IBM J. Res. Dev. 2001, 45, 47.

(34) Du, H.; Chen, C.; Krishnan, R.; Krauss, T. D.; Harbold, J. M.; Wise, F. W.; Thomas, M. G.; Silcox, J. Nano Lett. 2002, 2, 1321.

(35) Machol, J. L.; Wise, F.; Patel, R. C.; Tanner, D. B. Phys. Rev. B 1993, 48, 2819.

(36) Ai, X.; Guo, L.; Zou, Y.; Li, Q.; Zhu, H. Mater. Lett. 1999, 38, 131.

(37) Klein, M. C.; Hache, F.; Ricard, D.; Flytzanic, C. Phys. Rev. B 1990, 42,11123 .

(38) Efros, A. L.; Ekimov, A. I.; Kozlovskii, F.; Petrova-Koch, V.; Schmidbaur, H.; Sumilov, S. Solid State Commun. 1991, 78, 853.

(39) Efros, A. L.Electron-Hole Pair-Phonon Interaction in Semiconductor Nanocrystals. In Phonons in Semiconductor Nanostructures; Leburton, J.-P., Pascual, J., Sotomayor Torres, C., Eds.; NATO ASI Series; Kluwer Academic Publishers: Boston, 1993; Vol. 236, p 299.

(40) Schmitt-Rink, S.; Miller, D. A. B.; Chemla, D. C. Phys. Rev. B 1987, 35,8113 .

NL0502672 